\title{
Inpatient Management of Hospitalized Patients with Type 2 Diabetes
}

\author{
Andrew J. Ahmann, MD
}

\author{
Address \\ Oregon Health \& Science University, 318I SW Sam Jackson Park Road, \\ Portland, OR 97239-3098, USA. \\ E-mail: ahmanna@ohsu.edu \\ Current Diabetes Reports 2004, 4:346-35I \\ Current Science Inc. ISSN 1534-4827 \\ Copyright (? 2004 by Current Science Inc.
}

Over the past 4 years, the scientific literature addressing issues relevant to inpatient hyperglycemia and its management has grown dramatically but remains incomplete. The growing interest in inpatient diabetes management is particularly pertinent given the epidemic rise in the prevalence of type 2 diabetes and the associated increase in the proportion of inpatients carrying this diagnosis. The benefits of aggressive glucose control are well-established in certain admission categories. These benefits likely apply to many other admission diagnoses, but remain unproven at this time. Similarly, the best methods of glucose control remain uncertain in the various inpatient settings. Intensive insulin infusion therapy is becoming the standard care in the intensive care unit setting. Its use is also growing in less acute inpatient settings but requires further study. Inpatient subcutaneous insulin recommendations are general based on experience gained in the outpatient setting but offer a practical, physiologic approach.

\section{Introduction}

The management of hyperglycemia in the hospital, a therapeutic issue long ignored, has rather quickly arrived on the scene as a major area of new clinical information and quality improvement momentum. The medical literature is suddenly replete with documentation of the association between hyperglycemia and poor inpatient outcomes. Studies have demonstrated the benefit of intensive glucose control in the hospital and a comprehensive review on the management of diabetes and hyperglycemia was recently published $[1 \bullet \bullet]$. In response to this flurry of interest, professional organizations are promoting the generation of new guidelines for inpatient treatment of hyperglycemia that apply to all patients in the hospital [2•]. These guidelines resulted from a Consensus Conference sponsored by the American College of Endocrinology (ACE) with cosponsorship by the American Diabetes Association, the American Heart Association, the American Society of Anes- thesiologists, the Endocrine Society, the Society of Critical Care Medicine, the Society of Hospital Medicine, the Society of Thoracic Surgeons, and the American Association of Diabetes Educators. Hospitals and health care providers are now making efforts to develop effective systematic approaches to improve inpatient glucose control.

\section{Diabetes and Hyperglycemia in the Hospital}

It is not surprising that most of the recent literature on inpatient glucose issues has predominantly involved patients with type 2 diabetes, a chronic illness that is growing to epidemic proportions in the United States and throughout the world [3]. Accordingly, the population of diagnosed and previously undiagnosed type 2 diabetes will comprise an increasing percentage of hospitalized patients. In fact, the number of hospital discharge forms having diabetes listed as a diagnosis nearly doubled during the decade of the 1990s [4].

The exact proportion of hospitalized patients having a diagnosis of diabetes is not clear. This figure varies depending on such factors as the type of hospital (eg, community versus tertiary care referral hospital) and the demographics of the hospitals service area (eg, ethnic background of the community). Diabetes is common among patients admitted with a macrovascular diagnosis. For example, an international registry of 16,116 patients presenting with acute coronary syndrome in 14 countries reported a $25 \%$ prevalence of previously diagnosed diabetes [5]. This recent report did not give details on the overall frequency of hyperglycemia or the potential recognition of previously undiagnosed diabetes. Interestingly, the US sites reported a $31 \%$ prevalence of diabetes in patients with acute coronary syndrome, whereas other countries reported a much lower $22 \%$ prevalence.

Similarly, Gray et al. [6] recently reported their experience in 582 stroke patients in the United Kingdom. Sixtyeight percent of these patients had an admission blood glucose $\geq 110 \mathrm{mg} / \mathrm{dL}$. Fourteen percent had a previous diagnosis of diabetes. A subset of hyperglycemic patients without a previous diagnosis of diabetes returned for an oral glucose tolerance test 12 weeks later. Nearly $40 \%$ of these individuals had impaired glucose tolerance on the followup examination and $21 \%$ were found to have previously unrecognized diabetes. It was estimated that in addition to the $14 \%$ prevalence of known diabetes in patients present- 
ing with stroke, another $16 \%$ to $24 \%$ will have previously undiagnosed diabetes [6]. Nearly $30 \%$ will have impaired glucose tolerance. The authors also found that an admission blood glucose $\geq 110 \mathrm{mg} / \mathrm{d}$ combined with an admission hemoglobin $A_{1 c} \geq 6.2 \%$ was highly predictive of diabetes [6].

In a broad spectrum of patients hospitalized in a large community teaching hospital, Umpierrez et al. [7] found a $26 \%$ prevalence of diabetes, associated with increased morbidity and mortality. Another $12 \%$ of patients had blood glucose elevations greater than $200 \mathrm{mg} / \mathrm{dL}$. This finding of newly identified hyperglycemia without a known diagnosis of diabetes was also a strong predictor of adverse outcomes [7].

The direct consequences of hyperglycemia that lead to increased inpatient morbidity and death are far ranging $[1 \bullet \bullet, 8-10]$. Review of this topic is beyond the scope of this discussion. The general categories of adverse hyperglycemic consequences in the hospital include effects on immune function, vascular inflammatory processes, and thrombosis. Hyperglycemia-induced oxidative stress is a dominant theory underlying the proposed mechanism for the acute adverse events in this setting $[1 \bullet \bullet, 8]$.

\section{The Association Between Hyperglycemia and Adverse Hospital Outcomes}

Observational studies have demonstrated links between hyperglycemia and adverse outcomes for a wide variety of admission diagnoses $[1 \bullet \bullet]$. The most studied admission categories have been cardiovascular surgery, acute myocardial infarction, and surgical intensive care patients $[11,12,13 \bullet \bullet]$. Significant new information has been contributed in the areas of intensive care patients and cardiovascular surgery over the past 1 to 2 years, confirming and extending earlier findings for these and other patient groups.

\section{The impact of hyperglycemia on CABGS outcomes}

Furnary et al. [14] recently updated their previous large experience with patients undergoing coronary artery bypass graft surgery (CABGS). Their previous report had emphasized the dramatic reduction in deep sternal wound infections resulting from a more aggressive approach to glucose control in the 48 hours following surgery [12]. The updated report reviews data on 3554 CABGS patients carrying a diagnosis of diabetes (26\% of all CABGS patients from 1987 through 2001). After collecting detailed data on patients using a less intensive subcutaneous insulin strategy for several years, they found that the institution of an intravenous (IV) insulin infusion protocol appeared to decrease mortality among diabetic patients by $57 \%$ [14]. Their insulin infusion protocol has been intensified several times over the past decade.

Additionally, they reported a direct correlation between blood glucose elevations and mortality, particularly cardiacrelated mortality [14]. Mortality was $0.9 \%$ when the mean glucose over the first 48 hours following surgery was less than $150 \mathrm{mg} / \mathrm{dL}$, compared with a mortality of $14.5 \%$ when the mean glucose was greater than $250 \mathrm{mg} / \mathrm{dL}$. They did not report further improvement in outcomes with lower blood glucose levels. This analysis suffers somewhat because the period of inferior subcutaneous insulin treatment provided a disproportionate number of the patients experiencing glucose values at the highest levels $\left(e g_{1}>200 \mathrm{mg} / \mathrm{dL}\right)$. These patients were treated greater than 10 years before the final patients were included in the report, introducing the possible influence of other changes in surgical technique and postoperative care on the apparent relationship with blood glucose. Nevertheless, the data are quite convincing for the benefit of intensive glucose control in the perioperative and postoperative management of the CABG patient.

Furnary et al. [15] have now also reported a relationship between mean blood glucose and length of hospital stay (LOS). Reducing blood glucose by $50 \mathrm{mg} / \mathrm{dL}$ resulted in a mean reduction in LOS of approximately 1 day. They estimate the net cost savings from their intensive glucose control with an insulin infusion to be $\$ 5580$ [15].

A recent retrospective review by Estrada et al. [16], of 1574 patients undergoing CABGS between 1998 and 1999, strongly supports the findings of Furnary et al. [15]. Nearly $35 \%$ of the patients had a previous diagnosis of diabetes. Of the 1029 patients without a previous diagnosis of diabetes, over $50 \%$ experienced a mean blood glucose greater than $150 \mathrm{mg} / \mathrm{dL}$ during the day of and the day after surgery. No uniform method of postoperative glucose control had been proposed. Again, there was a direct relationship between glucose control and LOS as well as costs. A 50-mg/dL rise in glucose was associated with 0.76 additional hospitalization days in the hospital overall, and 0.99 additional hospital days for those with diabetes. This resulted in additional hospitalization costs of $\$ 2870$ per $50 \mathrm{mg} / \mathrm{dL}$ of glucose elevation for those with diabetes [16]. The correlation between mortality rate and hyperglycemia showed a strong trend that was not statistically significant in this analysis $(P=0.07)$.

A smaller, randomized prospective study of intensive IV insulin infusion versus "standard therapy" after CABGS was reported by Lazar et al. [17] this year. They treated 72 patients with insulin/dextrose/potassium infusions beginning at anesthetic induction and continued for 12 hours postoperatively. The standard group received subcutaneous regular insulin by "sliding scale," beginning at $4 \mathrm{U}$ for a blood glucose ranging between 250 to $299 \mathrm{mg} / \mathrm{dL}$. The mean glucose was approximately $130 \mathrm{mg} / \mathrm{dL}$ lower at 12 hours postoperatively ( $134 \mathrm{vs} 267 \mathrm{mg} / \mathrm{dL}$ ) in the insulin infusion group. The insulin infusion therapy was associated with a highly significant shorter LOS (mean difference of 2.7 days) and less atrial fibrillation. The more intensive therapy was also associated with a long-term survival advantage after 2 years $(P=0.04)$ and fewer recurrent wound infections [17]. Thus, this controlled trial confirmed the benefits of intensive glucose control on outcomes in diabetic patients undergoing CABGS. 


\section{The impact of hyperglycemia in the ICU}

To date, the single publication having the greatest impact on the advancement of inpatient therapy of hyperglycemia was that of Van den Berghe et al. [13••] 3 years ago. This study involved a relatively large population of patients $(n=1548)$ randomized to receive intensive IV insulin therapy (glucose goal $=80$ to $110 \mathrm{mg} / \mathrm{dL}$ ) or conventional therapy with a glucose target of 180 to $200 \mathrm{mg} / \mathrm{dL}$. The patients became eligible for the study once their glucose exceeded $110 \mathrm{mg} / \mathrm{dL}$. The dramatic results included reduction in intensive care unit (ICU) mortality by $42 \%$ and overall hospital mortality by $34 \%$. Reduced rates of sepsis, reduced time on the ventilator, and reduced requirement for dialysis were other significant benefits of aggressive glucose reduction. Although the lessons of this study almost certainly apply to patients with type 2 diabetes, one must keep in mind that only $13 \%$ of the included patients carried a diagnosis of diabetes. Furthermore, the implications are likely relevant to patients in medical ICUs as well as general medical and surgical floors, but the study itself included only surgical patients $(60 \%$ cardiac surgery patients) $[13 \bullet \bullet]$.

Very recently, Christiansen et al. [18] reported a small, prospective study of 135 very ill patients ( 97 surgical and 38 medical) cared for in a noncardiac multidisciplinary ICU over a 6-month period. Consistent with the report by Van den

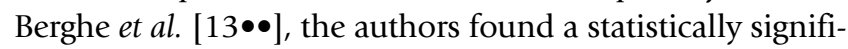
cant relationship between blood glucose and mortality, morbidity, and infection rates in the surgical group. The medical group showed similar trends that were not significant, probably due to the low statistical power of this analysis.

Finney et al. [19] also recently reported their experience with 523 ICU patients (mostly cardiac surgery patients) admitted over a 6 -month period. Their analytic methods were somewhat different, but confirmed a relationship between hyperglycemia and ICU mortality. Based on their data, the authors proposed a glucose target under $145 \mathrm{mg} /$ $\mathrm{dL}$ to improve ICU outcomes, a figure much more conservative than that of Van den Berghe et al. [13••]. Likewise, Laird et al. [20] also questioned the glucose goal of less than $110 \mathrm{mg} / \mathrm{dL}$ in an ICU population based on their findings in 516 trauma patients admitted to the ICU. They chose to evaluate three possible levels of early glycemic control as predictors of infection and mortality. Ninetyfour percent of these patients had a blood glucose over 110 $\mathrm{mg} / \mathrm{dL}$ and $60 \%$ had a blood glucose over $150 \mathrm{mg} / \mathrm{dL}$. On univariate analysis, mortality was statistically associated with blood glucose values $\geq 150 \mathrm{mg} / \mathrm{dL}$. However, on multivariate analysis, only a blood glucose $\geq 200 \mathrm{mg} / \mathrm{dL}$ was found to be an independent predictor of both infection and mortality [20].

Both the Finney et al. [19] and Laird et al. [20] studies support the relationship of hyperglycemia and increased morbidity and mortality in the ICU, but they come to different conclusions about the appropriate glucose goal of therapy. However, neither study is a prospective, interventional study and neither study has the scientific merit to change the conclusions of Van den Berghe et al. [13••]. Yet, it is not surprising that a recent survey of ICU physicians and nurses from five university-affiliated multidisciplinary ICUs reported significant variability in preferred targets for glucose control and concern about hypoglycemia [21].

Broadening the view of hyperglycemia in the hospital Although there is abundant observational and sound prospective interventional information on the relationship between hyperglycemia and adverse outcomes in cardiovascular surgery and the ICU, similar information applicable to the majority of hospitalized patients is lacking [22]. There is good reason to believe the same pathophysiologic principles will apply. Prospective, interventional studies are being performed in a variety of patient categories, including medical ICU patients and stroke patients $[23,24]$.

\section{Glucose Targets for Inpatient}

Treatment of Hyperglycemia

It is not easy to get consensus on standard targets for inpatient treatment of hyperglycemia. The ACE Inpatient Diabetes and Metabolic Control Consensus Conference convened to address this issue. After 2 days of expert presentation and debate, the ACE rendered the glycemic targets noted in Table $1[2 \bullet]$. These goals are quite ambitious and represent the ideal situation, largely based on Van den Berghe et al.'s [13••] respected work. Concerns center on the practicality and safety of these targets for all hospitals and all patients at this time. The American Diabetes Association and other cosponsors have not yet rendered their independent, full adoption of the ACE targets. Yet, the $\mathrm{ACE}$ and those involved in the Consensus Conference must be commended on their efforts. These are logical goals that challenge our hospitals and clinicians to develop safe and effective systems to attain them.

\section{Clinical Strategies to \\ Attain Inpatient Glucose Control}

No evidence-based guidelines are available to guide glucose management in all possible inpatient scenarios. Almost all of the recent research studies have used IV insulin infusions as the investigated intervention, often with comparator "standard therapy" that is poorly specified. When specified, the comparator usually introduces at least two variables, such as an alternative route of administration and an alternative treatment goal.

\section{The use of IV insulin infusions}

The previously mentioned prospective studies of CABG and surgical ICU patients implemented specific IV insulin protocols that consistently guided the investigators' attainment of the desired glucose targets $[13 \bullet \bullet, 15]$. Although these protocols clearly resulted in desirable outcomes, some clinicians have found them cumbersome, or for 
Table I. Goals for inpatient treatment of hyperglycemia

\begin{tabular}{|llll}
\hline & \multicolumn{2}{c|}{ Non-intensive care unit } & Labor and delivery \\
\cline { 2 - 3 } Intensive care unit & \multicolumn{1}{c|}{ Preprandial } & Maximal glucose & $\leq 100 \mathrm{mg} / \mathrm{dL}$ \\
\hline$\leq 110 \mathrm{mg} / \mathrm{dL}$ & $\leq 110 \mathrm{mg} / \mathrm{dL}$ & $\leq 180 \mathrm{mg} / \mathrm{dL}$ & \\
\hline (From Garber et al. $[2 \cdot])$. & & & \\
\hline
\end{tabular}

\section{Table 2. Proposed indications for intravenous insulin infusions}

\section{Definite}

Hyperglycemic emergencies (eg, diabetic ketoacidosis and hyperosmolar state)

Hyperglycemia in the critical care unit

Myocardial infarction or acute coronary syndrome

Perioperatively and postoperatively for heart surgery

NPO status for all patients with type I diabetes and insulin-deficient patients with type 2 diabetes

Labor and delivery if insulin is needed for glucose control

Probable

Perioperatively in all surgeries where glucose is elevated

Initial treatment of severe hyperglycemia associated with acute illness

Initial treatment for steroid-induced or exacerbated hyperglycemia

Organ transplantation, including pancreas transplantation

A dose-finding strategy to guide subcutaneous insulin in the hospital

NPO-nothing by mouth.

other reasons have chosen to explore other systematic options for IV insulin delivery. As a consequence, there are a number of published infusion protocols with demonstrated merit $[17,25 \bullet, 26-28]$. There is also the potential for a computer-assisted algorithm that would presumably reduce errors, continuously consider changes in insulin sensitivity and responsiveness, and reduce nursing time [29]. Some of the proposed indications for using IV insulin in the hospital are listed in Table 2.

The mechanisms by which insulin infusions improve outcomes are not absolutely clear at this point. Several investigators have argued that the benefit comes strictly from amelioration of the hyperglycemic state $[19,30]$. However, there is abundant scientific support for insulin infusions as favorable modulators of vascular reactivity, inflammatory cytokines, other inflammatory processes, and free fatty acid release $[1 \bullet \bullet, 9,10,31,32]$. Therefore, the benefits may go beyond the reduction in blood glucose.

The characteristics one would hope to find with an insulin infusion protocol include relatively rapid attainment of the goal glucose level, avoidance of excess hypoglycemia, and easy and consistent implementation by the nursing staff. The "Leuven" protocol (from Van den Berghe et al. $[13 \bullet \bullet])$ was associated with a $5.2 \%$ rate of hypoglycemia, compared with a $0.8 \%$ rate in the standard therapy group [30]. However, it must be noted that hypoglycemia was defined by a blood glucose of less than $40 \mathrm{mg} / \mathrm{dL}$, a much lower value than used by most other authors. They do not report the frequency of blood glucose below other cut-points, such as $60 \mathrm{mg} / \mathrm{dL}$. However, in trying to treat ICU patients with other proposed protocols, authors have shown only marginal improvements in the rate of hypoglycemia by the $40-\mathrm{mg} / \mathrm{dL}$ definition $[25 \bullet, 26]$. Apparently, the hypoglycemia that has been seen in any of the published studies has not had serious consequences.

In implementing an insulin infusion protocol, two factors that must not be forgotten are staff buy-in and adequate in-service training. Goldberg et al. [25•] failed in the effective implementation of the Leuven protocol. The clinical champions at their institution then developed an alternative protocol that was considered more "safe and clinically effective." However, in reviewing the authors' experience, it becomes apparent that the most critical factor in their successful second effort was not the new protocol but rather the improved process they used. They included more stakeholders in the development of the protocol, they employed extensive staff training, and they evaluated the success and staff satisfaction. These elements were apparently lacking in their initial rapid implementation of the Leuven protocol.

\section{Glycemic control beyond insulin infusions}

There is very little demonstrated or theoretic support for oral therapy in the hospital, considering the usual high acuity index of hospitalized patients where the inflexibility of oral agents becomes a liability $[1 \bullet \bullet]$. Of particular concern are the frequent contraindications to metformin therapy in this population and the growing concerns about issues involving exacerbation of congestive heart failure in those treated with thiazolidinediones [33-37]. Of course, there will always be exceptions where the continuation of outpatient oral agents is appropriate. 
Historically, subcutaneous insulin therapy for type 2 diabetes in the hospital has centered on "sliding scale" insulin. This practice has been appropriately condemned. Modern approaches emphasize the separate components of basal insulin, prandial, or nutritional insulin coverage, and then a correction component to promptly readjust for underestimates of requirements and for unpredictable variation $[1 \bullet \bullet, 27,38]$. These principles are largely predicated on the much broader experience with outpatient insulin therapy because no studies have systematically investigated various methods for administration of subcutaneous insulin in the hospital [39]. These principles appear reasonable and should improve the safety and efficacy of subcutaneous insulin therapy in the hospital.

The correction or supplemental rapid-acting insulin is typically added to baseline insulin therapy (combination of basal and nutritional insulin), but occasionally is added to oral agents or, rarely, is appropriate as stand-alone therapy. In any case, if this adjustable insulin is used routinely, one must increase the baseline insulin in a pattern indicated by the timing of the required corrections doses, intending to prevent a glucose rise in the future. Most supplemental insulin protocols offer several levels of insulin sensitivity with the appropriate level selected by the patient's previous response to insulin, body habitus, or concurrent factors affecting insulin response $(\mathrm{eg}$, steroid therapy or severe illness). The amount and pattern of correction insulin should be evaluated at least daily to make appropriate adjustments in both the baseline regimen and the sensitivity level for the correction scale.

When transitioning from an insulin infusion to subcutaneous insulin injections, the insulin infusion must overlap the initial subcutaneous injections of basal insulin by 1 to 3 hours, depending on the onset of action of the basal insulin (usually 3 hours for insulin glargine). The amount of basal insulin can be estimated from the insulin infusion rate in the last 4 to 12 hours before transition, extrapolated to a 24-hour total dose. If the insulin infusion covered just the basal requirements (plus a low-dose glucose infusion as a nutritional component), one may still be conservative using $80 \%$ of the calculated 24 -hour basal insulin requirement initially and then increasing the basal insulin daily as indicated by glucose monitoring four to seven times daily. If the patient was eating during the last 12 to 24 hours on the insulin infusion, one would calculate total daily insulin requirements, and reduce by $20 \%$. Then $50 \%$ to $60 \%$ of that dose is prescribed as daily basal insulin, with the remainder used to estimate the initial meal requirements divided into three portions. In some cases, carbohydrate counting can be used in the hospital to calculate the meal dose, but this will not be feasible in most settings. One can partially compensate for unpredictable food intake in the ill patient by dosing after the meal, converting fractions of the intended food intake into similar fractions of the anticipated full dose of rapid-acting (analogue) insulin. Regular insulin is not useful for such postprandial dosing.
Although most of the subcutaneous insulin principles noted earlier are derived from methods of treating type 1 diabetes, they are almost certainly appropriate for the inpatient management of type 2 diabetes. Frequent monitoring (at least before meals, bedtime, and probably once during the night) with multiple daily injections offers the opportunity for optimal correction and avoidance of hypoglycemia.

Virtually no evidence-based information is found in the literature for the initiation of subcutaneous insulin in the patient who did not previously receive IV insulin in the hospital or outpatient insulin prior to the admission. When one chooses to start subcutaneous insulin in this setting, it is probably safe to use a total daily dose of 0.6 to $0.8 \mathrm{U} / \mathrm{kg}$. One then defaults to the outpatient rule of approximately $50 \%$ of this total dose as basal insulin and the remainder as meal-related insulin, adjusted for the patient's actual intake. Additionally, one would initiate the supplemental insulin protocol to help guide baseline insulin adjustments for the individual patient.

\section{Conclusions}

It is time for all clinicians and all institutions to strive for improved quality of inpatient glucose control. This message is particularly important for the large and growing population of hospitalized patients with type 2 diabetes. In the past several years, much has been learned about the correlation between hyperglycemia and poor hospital outcomes for virtually every hospital admission diagnosis studied. Prospective studies have demonstrated the benefit of improved glucose control through the use of IV insulin infusion therapy to reduce infectious and vascular events in surgical ICU patients, patients with an acute myocardial infarction, and those having CABGS.

The lessons we have learned from these sound intervention studies are likely relevant to nearly all hospitalized patients, but broader confirmation is still indicated. We also must develop better, proven therapeutic protocols to cover the spectrum of patients at risk for hyperglycemic complications in the hospital, including systematic use of subcutaneous insulin injections. Eventually, technology, in the form of continuous glucose monitoring and computerassisted insulin infusions, will almost certainly advance the care of hospitalized patients.

\section{References and Recommended Reading}

Papers of particular interest, published recently, have been highlighted as:

- Of importance

-. Of major importance

$1 . \bullet$ Clement S, Braithwaite SS, Magee MF, et al.: Management of diabetes and hyperglycemia in hospitals. Diabetes Care 2004, 27:553-597.

A technical review of available research and developing clinical practices relevant to the inpatient management of hyperglycemia. Represents the most complete, well-referenced article on the topic. 
2. Garber AJ, Moghissi ES, Bransome ED Jr, et al.: American College of Endocrinology position statement on inpatient diabetes and metabolic control. Endocr Pract 2004, 10:77-82. The only published guidelines for inpatient treatment goals in diabetes. The culmination of a consensus conference of many stakeholders.

3. Department of Health and Human Services, Centers for Disease Control and Prevention: National Diabetes Fact Sheet. 2003. http://www.cdc.gov/diabetes/pubs/factsheet.htm. Accessed July 2004.

4. National Center for Chronic Disease Prevention and Health Promotion: Data \& Trends: Diabetes Surveillance System. http://www.cdc.gov/diabetes/statistics/index.htm. Accessed July 2004.

5. Franklin K, Goldberg RJ, Spencer F, et al.: Implications of diabetes in patients with acute coronary syndromes: the Global Registry of Acute Coronary Events. Arch Intern Med 2004, 164:1457-1463.

6. Gray CS, Scott JF, French JM, et al.: Prevalence and prediction of unrecognised diabetes mellitus and impaired glucose tolerance following acute stroke. Age Ageing 2004, 33:71-77.

7. Umpierrez GE, Isaacs SD, Bazargan N, et al.: Hyperglycemia: an independent marker of in-hospital mortality in patients with undiagnosed diabetes. J Clin Endocrinol Metab 2002, 87:978-982.

8. Le Roith D: Molecular mechanisms by which metabolic control may improve outcomes. Endocr Pract 2004, 10(suppl 2):57-62.

9. Marik PE, Raghavan M: Stress-hyperglycemia, insulin and immunomodulation in sepsis. Intensive Care Med 2004, 30:748-756.

10. Andersen SK, Gjedsted J, Christiansen C, Tonnesen E: The roles of insulin and hyperglycemia in sepsis pathogenesis. J Leukoc Biol 2004, 75:413-421.

11. Malmberg K: Prospective randomised study of intensive insulin treatment on long term survival after acute myocardial infarction in patients with diabetes mellitus. DIGAMI (Diabetes Mellitus, Insulin Glucose Infusion in Acute Myocardial Infarction) Study Group. BMJ 1997, 314:1512-1515.

12. Furnary AP, Zerr KJ, Grunkemeier GL, Starr A: Continuous intravenous insulin infusion reduces the incidence of deep sternal wound infection in diabetic patients after cardiac surgical procedures. Ann Thorac Surg 1999, 67:352-360; discussion 360-362.

13. $\bullet$ Van den Berghe GP, Wouters P, Weekers F, et al.: Intensive insulin therapy in the critically ill patients. $N$ Engl J Med 2001, 345:1359-1367.

A prospective study of 1548 adult patients in the surgical ICU, randomized to intensive insulin infusion therapy or standard treatment. A strategy to maintain blood glucose under $110 \mathrm{mg} / \mathrm{dL}$ resulted in a dramatic reduction in mortality, sepsis, and other parameters of morbidity

14. Furnary AP, Gao G, Grunkemeier GL, et al:: Continuous insulin infusion reduces mortality in patients with diabetes undergoing coronary artery bypass grafting. J Thorac Cardiovasc Surg 2003, 125:1007-1021.

15. Furnary AP, Wu Y, Bookin SO: Effect of hyperglycemia and continuous intravenous insulin infusions on outcomes of cardiac surgical procedures: the Portland Diabetic Project. Endocr Pract 2004, 10(suppl 2):21-33.

16. Estrada CA, Young JA, Nifong LW, Chitwood WR Jr: Outcomes and perioperative hyperglycemia in patients with or without diabetes mellitus undergoing coronary artery bypass grafting. Ann Thorac Surg 2003, 75:1392-1399.

17. Lazar HL, Chipkin SR, Fitzgerald CA, et al.: Tight glycemic control in diabetic coronary artery bypass graft patients improves perioperative outcomes and decreases recurrent ischemic events. Circulation 2004, 109:1497-1502.

18. Christiansen C, Toft P, Jorgensen HS, et al.: Hyperglycaemia and mortality in critically ill patients: a prospective study. Intensive Care Med 2004, 30:1685-1688.
19. Finney SJ, Zekveld C, Elia A, Evans TW: Glucose control and mortality in critically ill patients. JAMA 2003, 290:2041-2047.

20. Laird AM, Miller PR, Kilgo PD, et al: : Relationship of early hyperglycemia to mortality in trauma patients. J Trauma 2004, 56:1058-1062.

21. McMullin J, Brozek J, Jaeschke R, et al:: Glycemic control in the ICU: a multicenter survey. Intensive Care Med 2004, 30:798-803.

22. Coursin DB, Connery LE, Ketzler JT: Perioperative diabetic and hyperglycemic management issues. Crit Care Med 2004 32(4 suppl):S116-S125.

23. Van den Berghe GH: Role of intravenous insulin therapy in critically ill patients. Endocr Pract 2004, 10(suppl 2):17-20.

24. Gray CS, Hildreth AJ, Alberti GK, et al.: Poststroke hyperglycemia: natural history and immediate management. Stroke 2004, 35:122-126.

25. Goldberg PA, Siegel MD, Sherwin RS, et al.: Implementation of a safe and effective insulin infusion protocol in a medical intensive care unit. Diabetes Care 2004, 27:461-467.

A very recent demonstration of process issues in developing a

successful inpatient protocol.

26. Kanji S, Singh A, Tierney M, et al.: Standardization of intravenous insulin therapy improves the efficiency and safety of blood glucose control in critically ill adults. Intensive Care Med 2004, 30:804-810.

27. Trence DL, Kelly JL, Hirsch IB: The rationale and management of hyperglycemia for in-patients with cardiovascular disease: time for change. J Clin Endocrinol Metab 2003, 88:2430-2437.

28. Markovitz LJ, Wiechmann RJ, Harris N, et al.: Description and evaluation of a glycemic management protocol for patients with diabetes undergoing heart surgery. Endocr Pract 2002, 8:10-18.

29. Bode BW, Braithwaite SS, Steed RD, Davidson PC: Intravenous insulin infusion therapy: indications, methods, and transition to subcutaneous insulin therapy. Endocr Pract 2004, 10(suppl 2):71-80.

30. Van den Berghe G, Wouters PJ, Bouillon R, et al:: Outcome benefit of intensive insulin therapy in the critically ill: Insulin dose versus glycemic control. Crit Care Med 2003, 31:359-366.

31. Dandona P, Aljada A, Bandyopadhyay A: The potential therapeutic role of insulin in acute myocardial infarction in patients admitted to intensive care and in those with unspecified hyperglycemia. Diabetes Care 2003, 26:516-519.

32. Hirsch IB: Effect of insulin therapy on nonglycemic variables during acute illness. Endocr Pract 2004, 10(suppl 2):63-70.

33. Calabrese AT, Coley KC, DaPos SV, et al:: Evaluation of prescribing practices: risk of lactic acidosis with metformin therapy. Arch Intern Med 2002, 162:434-437.

34. Emslie-Smith AM, Boyle DI, Evans JM, et al.: Contraindications to metformin therapy in patients with type 2 diabetesa population-based study of adherence to prescribing guidelines. Diabet Med 2001, 18:483-488.

35. Masoudi FA, Wang Y, Inzucchi SE, et al:: Metformin and thiazolidinedione use in Medicare patients with heart failure. JAMA 2003, 290:81-85.

36. Nesto RW, Bell D, Bonow RO, et al:: Thiazolidinedione use, fluid retention, and congestive heart failure: a consensus statement from the American Heart Association and American Diabetes Association. October 7, 2003. Circulation 2003, 108:2941-2948.

37. Mudaliar S, Chang AR, Henry RR: Thiazolidinediones, peripheral edema, and type 2 diabetes: incidence, pathophysiology, and clinical implications. Endocr Pract 2003, 9:406-416.

38. Magee MF, Clement S: Subcutaneous insulin therapy in the hospital setting: issues, concerns, and implementation. Endocr Pract 2004, 10(suppl 2):81-88.

39. DeWitt DE, Hirsch IB: Outpatient insulin therapy in type 1 and type 2 diabetes mellitus: scientific review. JAMA 2003, 289:2254-2264. 